\title{
Adaptación transcultural del cuestionario POSAS (Patient and Observer Scar Assessment) para valoración de cicatrices
}

\author{
Tomás Rodríguez C. ${ }^{1}$, Antonella Sanguineti M. ${ }^{1}$, Natalia Moreno B. ${ }^{1}$, \\ Katya Carrillo G. ${ }^{1}$, Andrea Hasbún N. ${ }^{2}$ y Sebastián López N. ${ }^{1}$
}

\section{Transcultural adaptation of the POSAS questionnaire (Patient and Observer Scar Assessment) to assess scars}

\begin{abstract}
Introduction: The POSAS scale for the evaluation of scars is validated and shows advantages over other scales by integrating qualitative parameters and the opinion of the patient. It has a growing use in clinical practice, but its transcultural adaptation is needed for its local application. Aim: To carry out a transcultural adaptation of the original POSAS 2.0 scale. Materials and Method: With dynamic translation technique, translation of POSAS in english, comparison of translations, inverse translation and pilot test in observers and patients. Results: Good correlation of translations was obtained, observers and patients were able to understand the instrument adapted in a pilot study. Conclusion: We obtained an adapted version of the POSAS scale, applicable to the local population for assessment of different types of scars. The adapted scale can be submitted to validation processes.

Key words: POSAS; translation; adaptation; spanish.
\end{abstract}

\section{Resumen}

Introducción: La escala POSAS para evaluación de cicatrices está validada y muestra ventajas sobre otras escalas por integrar parámetros cualitativos y la opinión del paciente. Tiene un uso creciente en la práctica clínica, pero hace falta su adaptación transcultural para su aplicación local. Objetivo: Realizar una adaptación transcultural de la escala POSAS 2.0 original. Materiales y Método: Con técnica de traducción dinámica se efectuó traducción de POSAS en inglés, comparación de traducciones, traducción inversa y prueba piloto en observadores y pacientes. Resultados: Se obtuvo buena correlación de traducciones, los observadores y pacientes lograron comprender el instrumento adaptado en un estudio piloto. Conclusión: Obtuvimos una versión adaptada de la escala POSAS, aplicable a población local para valoración de diferentes tipos de cicatrices. Se puede someter la escala adaptada a procesos de validación.

Palabras clave: POSAS; traducción; adaptación; español.

\section{Introducción}

Las cicatrices son el resultado invariable de quemaduras, traumas y procedimientos quirúrgicos. El manejo de las cicatrices puede ser complejo $\mathrm{y}$ requiere una evaluación objetiva que permita cuantificar o calificar la cicatriz para evaluar sus cambios y medir respuesta a los tratamientos ${ }^{1}$. Se han desarrollado múltiples instrumentos para la evaluación de las cicatrices que incluyen parámetros objetivos y subjetivos. Las mediciones objetivas son cuantitativas y estandarizadas, y requieren de aparatos que miden los atributos físicos de la cicatriz. Las mediciones subjetivas dependen de uno o más observadores y aportan información cualitativa. Hay métodos semicuantitativos como las escalas de evaluación, para transformar la evaluación subjetiva en una medición más estandarizada.

Las principales características físicas medibles en una cicatriz son su flexibilidad, firmeza, color, grosor, perfusión y superficie, que en conjunto reflejan procesos tisulares en la cicatriz ${ }^{2}$. Algunas
'Departamento de Cirugía, Hospital Clínico Universidad de Chile. Santiago, Chile. ¿Unidad de Cirugía Plástica. Hospital de Niños Roberto del Río. Santiago, Chile.

Recibido el 8 de diciembre de 2018 y aceptado para publicación el 7 de enero de 2019

Correspondencia a: Dr. Tomás Rodríguez C. dr.to.rodriguez@gmail.com 
herramientas de medición objetiva para estos parámetros son el pneumatonómetro y el cutómetro para la flexibilidad (aplican presión a la cicatriz), el durómetro (que aplica una carga en la cicatriz), análisis colorimétrico con espectrofotómetro, ecografía cutánea (TUPS: tissue ultrasound palpation system) para evaluar el grosor, láser doppler para evaluar la microperfusión en las quemaduras y la topografía tridimensional entre otras técnicas ${ }^{3-5}$. Todas ellas han demostrado una aceptable correlación con los parámetros a medir, sin embargo, tienen alto costo y requieren una curva de aprendizaje para emplearse. Estas herramientas actualmente son consideradas el "gold standard" para la medición física de las cicatrices, sin embargo, hasta el momento no existe consenso sobre su real beneficio ${ }^{6}$.

Entre las herramientas subjetivas para medición de las cicatrices, las escalas han cobrado relevancia por su adecuada correlación con los parámetros objetivos y el menor costo de implementación. Las escalas de valoración de cicatrices más utilizadas son:

- Vancouver Scar Scale (VSS).

- Visual Analog Scale with Scar Ranking (VASSR).

- Patient and Observer Scar Assessment Scale (POSAS).

- Manchester Scar Scale (MSS).

- The Stony Brook Scar Evaluation Scale (SBSES).

Si bien estas escalas se han validado en consistencia y son herramientas confiables, la mayoría utiliza parámetros de evaluación con datos categóricos u ordinales con pocos niveles, lo que les da capacidad de reflejar diferencias mayores entre las cicatrices, pero son deficientes para evaluar diferencias finas. En todas estas escalas se suma un puntaje total, sin diferenciar por ítem, por lo que cicatrices muy diferentes podrían tener el mismo puntaje. La mayoría no considera los síntomas asociados a la cicatriz como el dolor y el prurito ni tampoco el impacto funcional para el paciente, aspecto que cada vez cobra mayor relevancia en la evaluación de condiciones crónicas. Las características de las escalas mencionadas se enlistan en la Tabla 1. De estas escalas destaca POSAS (Patient and Observer Scar Assessment) ya que considera la opinión del paciente y, además, evalúa los síntomas asociados.

Si bien POSAS no está diseñada para evaluar funcionalidad ni calidad de vida, es una escala que abarca elementos cualitativos, sin dejar de ofrecer un uso cuantitativo de la herramienta.

\section{POSAS original}

Esta escala se desarrolló por primera vez en el año 2005, por un grupo de investigadores alemanes, que han realizado hasta la fecha una actualización (POSAS 2.0) en versión alemán e inglés. POSAS ha sido sometido a estudios de validez (confiabilidad, consistencia interna, validez de contenido, validez concurrente, factibilidad en clínica). Ha sido utilizada para evaluar todo tipo de cicatrices (lineales, quemaduras, queloídeas) con buena correlación con los estudios clinométricos. Las pruebas con pacientes han demostrado que un sólo observador es suficiente para una evaluación confiable, sin embargo, más observadores mejoran la confiabilidad ${ }^{7,8}$.

POSAS requiere sensación táctil, por lo que no se utiliza en evaluación de las cicatrices con fotografías. Es una herramienta que no requiere entrenamiento pudiendo ser utilizada en todo tipo de cicatrices.

El instrumento consta de dos escalas, una para evaluación del observador y otra para evaluación del paciente, siendo ambas complementarias. Idealmente ambas escalas deben aplicarse en la misma consulta, sin embargo, se pueden aplicar por separado si fuese necesario. El paciente puede realizar su evaluación donde se sienta más cómodo y luego enviar las respuestas de forma remota a su tratante, ya que es una herramienta simple y diseñada para autollenado.

\section{Tabla 1. Resumen de escalas de valoración de cicatrices}

\begin{tabular}{|ll|}
\hline VSS & $\begin{array}{l}\text { Es ampliamente utilizada, sin embargo, tiene algunas desventajas como basar su evaluación solo en la observación, no considera la } \\
\text { evaluación del paciente ni los síntomas como prurito y dolor. Mide } 4 \text { parámetros (vascularidad, grosor, flexibilidad y pigmentación) } \\
\text { en un rango de } 0-13 \text { puntos }\end{array}$ \\
VASSR & $\begin{array}{l}\text { Es una escala basada en la observación de una fotografía de la cicatriz, evalúa } 4 \text { parámetros (pigmentación, vascularidad, acepta- } \\
\text { bilidad y confort del observador) más contorno. Simple y con buena correlación respecto a opinión de expertos, sin embargo, poco } \\
\text { aplicable en otros contextos }\end{array}$ \\
MSS & Bastante completa ya que mide 7 parámetros, sin embargo, aún no es utilizada ampliamente como las otras escalas \\
SBSES & Mide 6 parámetros, usa datos binarios (0-1) lo que limita su sensibilidad para encontrar diferencias entre cicatrices \\
\hline
\end{tabular}




\section{Escala del observador}

Consta de seis parámetros a evaluar en una escala ordinal de 1 a 10. La suma de todas las preguntas da un puntaje final (6 a 60 puntos). Además, se incluye una pregunta sobre opinión global de la cicatriz que no se suma al puntaje. Los cuadros de categoría aportan una evaluación cualitativa más completa y tampoco se incluyen en el cálculo del puntaje.

\section{Escala del paciente}

Consta de 7 preguntas: 2 exploran síntomas (dolor y prurito), 4 exploran parámetros (color, rigidez, grosor, superficie) y 1 sobre opinión global de la cicatriz. Todas se evalúan en escala ordinal de 1 a 10. La suma de todas las preguntas da un puntaje final (7 a 70 puntos).

Esta encuesta es ampliamente utilizada en la práctica clínica, sin embargo, por encontrarse en idioma inglés se limita su uso en población local. Es necesario contar con una versión adaptada para aplicarla en nuestro contexto.

Este trabajo tiene como objetivo obtener una versión adaptada transculturalmente de la escala POSAS para su validación y aplicación en población local.

\section{Materiales y Método}

Se obtiene autorización de los autores originales de POSAS y POSAS 2.0 para realizar la adaptación transcultural de encuesta original en inglés al español chileno. Se realiza traducción al español por un traductor A (persona con lengua materna inglesa que ha estado en Chile por más de 10 años) y un traductor B (cuyo idioma materno es el español, que ha vivido en Chile los últimos 10 años y tiene certificado de dominio avanzado del inglés). Estas traducciones son evaluadas por un comité revisor quienes realizan cambios para así obtener una versión provisoria del español chileno de la encuesta POSAS (Figura 1). Utilizando la técnica de traducción dinámica se pretende que la traducción no pierda su naturaleza original respetando las palabras e idea gramatical.

La versión provisoria en español chileno se somete a traducción inversa al idioma inglés por un traductor $\mathrm{C}$ (lengua nativa inglés, que ha vivido más de 15 años en Chile) la que es comparada con la versión original buscando concordancia entre ambas. En caso de ser necesario (falta de concordancia y equivalencia), se realiza cambios en versión provisoria español chileno para poder obtener una versión final adaptada al español chileno la que será sometida a una prueba piloto aplicando este instrumento a un grupo de observadores y pacientes, evaluando comprensión lingüística y dificultades. Se excluye de prueba piloto a pacientes sin consentimiento informado (Figura 2).

\section{Resultados}

Luego de obtener autorización de los autores originales de POSAS y POSAS 2.0 en inglés para realizar la adaptación transcultural, se realizó una traducción al español chileno por un traductor A (médico fisiatra, de lengua materna inglesa estadounidense, que ha vivido más de 10 años en Chile) y un traductor B (cirujano general, de lengua nativa español chileno con certificado de dominio avanzado del inglés). Se realizó una comparación de traducciones en un grupo de cinco personas (equipo coloproctología Hospital Clínico Universidad de

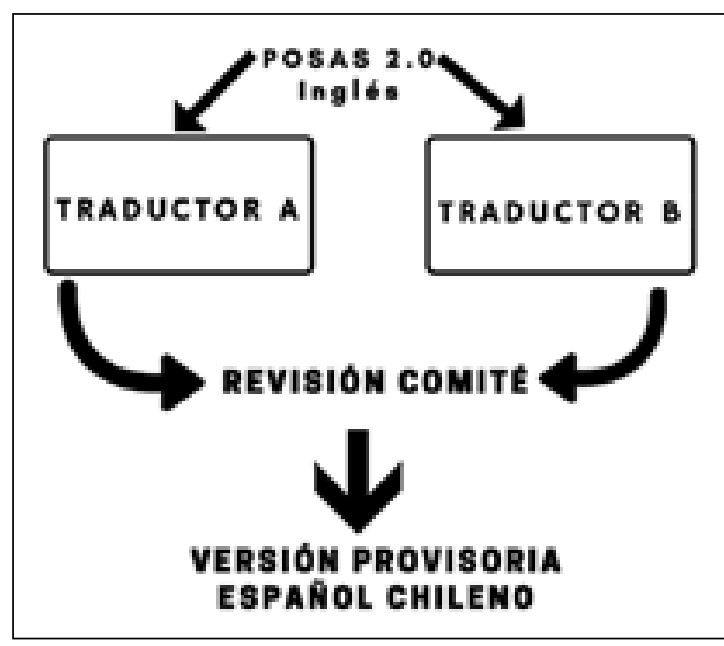

Figura 1. Rol del Comité de Revisión.

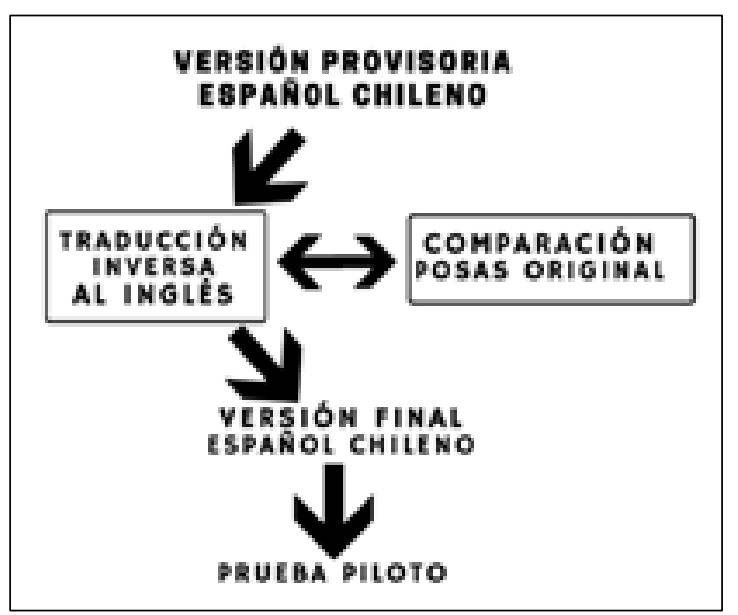

Figura 2. 
Chile, excluyendo a los traductores A y B) llegando a una versión única. Cuando hubo discordancia entre traducciones, el grupo decidió en conjunto las palabras o frases más adecuada, en el contexto de la escala, obteniendo así la versión provisoria del español chileno de la encuesta POSAS (Figura 1).

\section{Traducción inversa}

Una vez obtenida la versión provisoria del español chileno, esta se somete a traducción inversa al idioma inglés, por un traductor $\mathrm{C}$ (cardiocirujano, lengua nativa inglés, que ha vivido más de 15 años en Chile). La versión inversa fue comparada con la versión original evaluando concordancia entre ambas. La comparación mostró alta concordancia y equivalencia por lo que no se realizan modificaciones a versión provisoria en español chileno. De esta forma se tiene una versión final adaptada al español chileno que logra concordancia con POSAS original (Figura 2).

\section{Prueba piloto}

Se aplica la versión provisoria adaptada al español chileno a un grupo de observadores y pacientes en un estudio piloto para evaluar comprensión lingüística y factibilidad de aplicación en clínica. Se aplicó el instrumento adaptado a un grupo de 60 personas (30 pacientes y 30 observadores) que acuden a consulta ambulatoria de cirugía plástica y cirugía general en el Hospital Roberto del Río y Hospital Clínico de la Universidad de Chile. Se realizan encuestas individuales. Se seleccionan pacientes que presenten cicatrices de diferentes formas, extensiones y zonas del cuerpo, de los cuales 28 son posoperatorias y 2 como secuela de fasceitis necrotizante. Dos de los pacientes evaluados eran menores de 18 años y en estos casos se realiza la encuesta al paciente acompañados de la madre.

Se obtuvo respuestas satisfactorias en todas las encuestas realizadas, tanto a los observadores como a los pacientes, sin presentar dificultades de comprensión.

En todos los casos las encuestas fueron respondidas en menos de 8 minutos.

\section{Discusión}

La evaluación de la evolución clínica de las cicatrices es importante, ya que es una forma objetiva de evaluación de resultados clínicos, siendo un estándar de cuidado en medicina. Un buen instrumento de evaluación de cicatrices debe incluir no solo las características físicas de una cicatriz, sino también debe contemplar síntomas asociados idealmente con participación directa del paciente tal como lo contempla la escala POSAS. Estos instrumentos deben tener la consistencia y confiabilidad adecuadas para ser considerados fiables y válidos. POSAS ha sido sometido a estudios de validez (confiabilidad, consistencia interna, validez de contenido, validez concurrente, factibilidad en clínica) y ha sido utilizada para evaluar todo tipo de cicatrices con buena correlación con los estudios clinométricos 9 .

Existen estudios y revisiones donde se comparan los instrumentos semicuantitativos para evaluación de cicatrices, demostrando que la escala de evaluación de cicatrices de Vancouver es la más frecuentemente utilizada, sin embargo, la escala POSAS parece ser la más completa teniendo en cuenta la opinión del paciente ${ }^{10}$. Otras revisiones demuestran que POSAS es más confiable y exacta para evaluar cicatrices que VSS ${ }^{11,12}$.

Para aplicar una herramienta como la escala POSAS en una población determinada es necesario que ésta se traduzca y adapte a la población, no siendo intercambiables por otras traducciones en español dadas las múltiples diferencias socioculturales y lingüísticas, producto de un proceso dinámico de cambio inter e intrapoblacional. Las escalas siguen siendo útiles, pero se debe realizar una adaptación transcultural cuidadosa para no cambiar su significado original, conservando la equivalencia de los elementos entre las culturas ${ }^{13}$. En este trabajo, aplicando técnicas de traducción dinámica, obtuvimos un cuestionario adaptado a los chilenos que está disponible para completar su validación mediante estudios con población local (ver archivo adjunto con cuestionarios para observador y paciente).

\section{Conclusión}

Obtuvimos una versión adaptada transculturalmente del cuestionario POSAS (Patient and Observer Scar Assessment) para valoración de cicatrices para ser utilizada en población chilena. Esta versión adaptada puede iniciar un proceso de validación con población local.

\section{Responsabilidades éticas}

Protección de personas y animales. Los autores declaran que para esta investigación no se han realizado experimentos en seres humanos ni en animales.

Confidencialidad de los datos. Los autores declaran que en este artículo no aparecen datos de pacientes.

Conflictos de interés: no hay. 


\section{Archivos adjuntos}

Escala POSAS del observador

The Patient and Observer Scar Assessment Scale v 2.0 / EN

Fecha del examen:

Nombre del paciente:

Observador:

Centro:

Fecha de nacimiento del paciente:

Investigación clínica:

RUT/ficha del paciente:

\section{Marque en el esquema la cicatriz a evaluar}
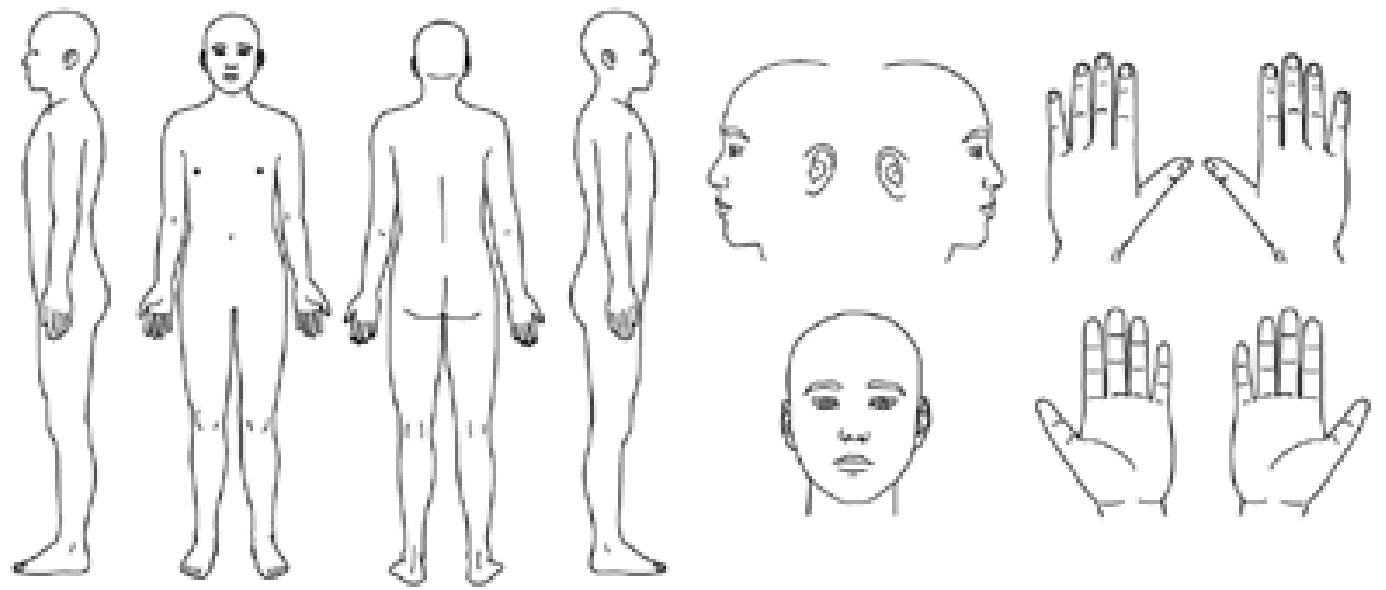

Califique cada ítem del 1 al 10:

$1=$ Similar a piel normal $\quad 10=$ Muy diferente a piel normal

\begin{tabular}{|l|l|l|l|l|l|l|l|l|l|l|l|}
\hline Parámetro & $\mathbf{1}$ & $\mathbf{2}$ & $\mathbf{3}$ & $\mathbf{4}$ & $\mathbf{5}$ & $\mathbf{6}$ & $\mathbf{7}$ & $\mathbf{8}$ & $\mathbf{9}$ & $\mathbf{1 0}$ & Categoría (comparar con piel normal circundante) \\
\hline Vascularidad & & & & & & & & & & & Pálido / Rosado / Rojo / Morado / Mixto \\
\hline Pigmentación & & & & & & & & & & & Hipo / Hiper / Mixto \\
\hline Elevación & & & & & & & & & & & Gruesa / Adelgazada \\
\hline Rugosidad & & & & & & & & & & & Más / Menos / Mixto \\
\hline Flexibilidad & & & & & & & & & & & Flexible / Rígido / Mixto \\
\hline Área de superficie & & & & & & & & & & & Expansión / Retracción / Mixto \\
\hline Opinión general & & & & & & & & & & & \\
\hline
\end{tabular}

\section{Explicación}

La escala del observador de POSAS consiste en 6 ítems (vascularidad, pigmentación, elevación, rugosidad, flexibilidad, área de superficie). Todos los ítems son calificados en una escala con rango de 1 ("similar a piel normal") a 10 ("Muy diferente a piel normal").

La suma de los 6 ítems resulta en un Score total de la "Escala del Observador del POSAS". A cada ítem se agregan categorías. Además, una opinión general es calificada en una escala de 1 a 10. Todos los parámetros deben preferentemente ser comparados a la piel normal del paciente en una ubicación anatómica comparable.

Notas explicativas para los ítems:

- Vascularidad: Presencia de vasos en la cicatriz, evaluado por el enrojecimiento, probado por el retorno sanguíneo tras la vitropresión

- Pigmentación: Coloración oscura de la cicatriz por pigmento (melanina); aplicar vitropresión moderada para eliminar el efecto de la vascularidad.

- Elevación: Altura de la cicatriz respecto a la piel normal circundante.

- Rugosidad: Irregularidad en la superficie de la cicatriz.

- Flexibilidad: Elasticidad de la cicatriz evaluada al arrugarla entre los dedos pulgar e índice.

- Área de superficie: Área de la cicatriz en relación al área de la herida original. 


\section{ARTÍCULO ORIGINAL}

Escala POSAS del paciente

The Patient and Observer Scar Assessment Scale v 2.0 / EN

Fecha del examen:

Nombre del paciente:

Observador:

Centro:

Fecha de nacimiento del paciente:

Investigación clínica:

RUT/ficha del paciente:

\section{Marque en el esquema la cicatriz a evaluar}

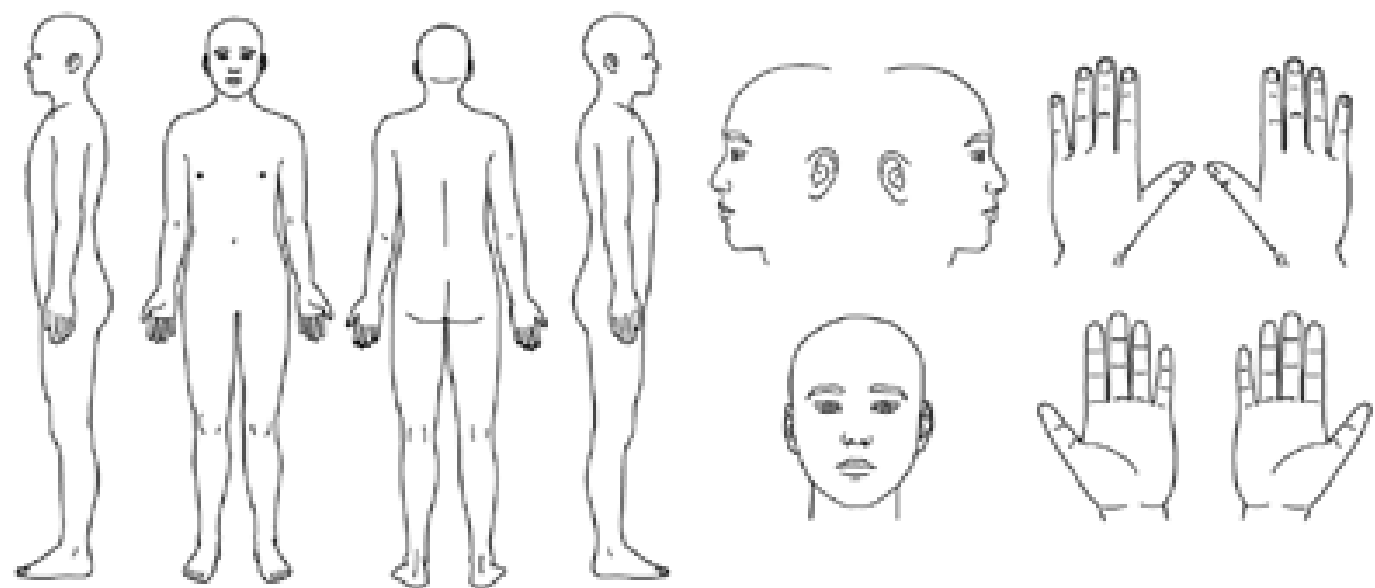

Califique cada ítem del 1 al 10:

\begin{tabular}{|c|c|c|c|c|c|c|c|c|c|c|}
\hline & & & & & & & & & 0 & 10 \\
\hline \multicolumn{11}{|l|}{ ¿Ha sido la cicatriz dolorosa en las últimas semanas? } \\
\hline ¿Ha estado con picazón en la cicatriz en las últimas semanas? & & & & & & & & & & \\
\hline
\end{tabular}

$1=$ No, como piel normal $10=$ Sí, muy diferente

\begin{tabular}{|c|c|c|c|c|c|c|c|c|c|c|}
\hline & 1 & 2 & 3 & 4 & 5 & 6 & 7 & 8 & 9 & 10 \\
\hline ¿Es la elevación de la cicatriz distinta a la piel normal actu & & & & & & & & & & \\
\hline ¿Es la cicatriz más rugosa que la piel normal actualmente? & & & & & & & & & & \\
\hline
\end{tabular}

$1=$ Como piel normal $\quad 10=$ Muy diferente

\begin{tabular}{|c|c|c|c|c|c|c|c|c|c|c|}
\hline & 1 & 2 & 3 & 4 & 5 & 6 & 7 & 8 & 9 & 10 \\
\hline ¿Cuál es su opinión general de la cicatriz comparada con la piel normal? & & & & & & & & & & \\
\hline
\end{tabular}




\section{Bibliografía}

1. Van de Kar A, Corion L, Smeulders M, Draaijers L, Van der Horst L, Van Zuijlen P. Evaluación confiable y factible de cicatrices lineales por parte de la Escala de evaluación de cicatrices de pacientes y observadores. Plast Reconstr Surg. 2005; 116:514-22.

2. Andrades P, Benítez S, Prado A. Guidelines for the treatment of kelloids and hipertrophic scars. Rev Chil Cir. 2016;58:78-88

3. Lee K, Dretzke J, Grover L, Logan A Moiemen N. A systematic review of objective burn scar measurements. Burns Trauma 2016;4:14.

4. Ud-Din S, Bayat A. Non-invasive objective devices for monitoring the inflammatory, proliferative and remodelling phases of cutaneous wound healing and skin scarring. Exp Dermatol. 2016;25:579-85.

5. Perry D, McGrouther D, Bayat A.
Current tools for noninvasive objective assessment of skin scars. Plast Reconstr Surg. 2010;126:912-23.

6. Leyva-Gómez G, Zacáula N, Álvarez M, Tolentino K, Morales M. Evaluación de las propiedades biomecánicas de la piel en un paciente quemado con un método no invasivo y cuantitativo. Rev Investig Discap. 2017;6:88-93.

7. Fearmonti R, Bond J, Erdmann D, Levinson $\mathrm{H}$. A review of scar scales and scar measuring devices. Eplasty. 2010;10:e43.

8. Tyack Z, Simons M, Spinks A, Wasiak J. A systematic review of the quality of burn scar rating scales for clinical and research use. Burns 2012;38:6-18.

9. Truong P, Lee J, Soer B, Gaul C, Olivotto I. Reliability and validity testing of the Patient and Observer Scar Assessment Scale in evaluating linear scars after breast cancer surgery. Plast Reconstr Surg. 2007;119:487-94.
10. Vercelli S, Ferriero G, Sartorio F, Stissi $\mathrm{V}$, Franchignoni F. How to assess postsurgical scars: a review of outcome measures. Disability and Rehabilitation. 2009;31:2055-63.

11. Draaijers J, Tempelman F, Botman Y, Tuinebreijer W , Middelkoop E, Kreis $\mathrm{R}$, et al. La escala de evaluación de cicatrices de pacientes y observadores: una herramienta confiable y viable para la evaluación de cicatrices. Plast Reconstr Surg. 2004;113:1960-5.

12. Falder S, Browne A, Edgar D, Staples E, Fong J, Rea S, et al. Outcomes for adult burn survivors: a clinical overview. Burns 2009;35:61841.

13. Calvo O, Oliveros R, Sánchez R. Adaptación cultural del formulario EORTC QLQ CR-29 para su aplicación en pacientes con cáncer de recto en el Instituto Nacional de Cancerología de Colombia. Rev Colomb Cancerol. 2010;14;189-98. 